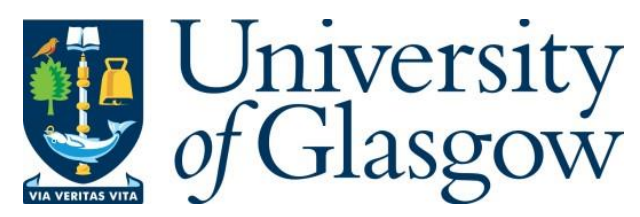

Macdonald, S., Morrison, J., Melville, C.A., Baltzer, M., MacArthur, L. and Cooper, S. A. (2018) Embedding routine health checks for adults with intellectual disabilities in primary care: practice nurse perceptions. Journal of Intellectual Disability Research, 62(4), pp. 349-357. (doi:10.1111/jir.12475)

There may be differences between this version and the published version. You are advised to consult the publisher's version if you wish to cite from it.

http://eprints.gla.ac.uk/157596/

Deposited on: 17 April 2018

Enlighten - Research publications by members of the University of Glasgow http://eprints.gla.ac.uk 


\section{Embedding routine health checks for adults with intellectual disabilities in primary care: practice nurse perceptions.}

Abstract

\section{Background}

Adults with intellectual disabilities have consistently poorer health outcomes than the general population. There is evidence that routine health checks in primary care may improve outcomes. We conducted a randomised controlled trial of practice nurse led health checks. Here we report findings from the nested qualitative study.

\section{Aim}

To explore practice nurse perceptions and experience of delivering an anticipatory health check for adults with intellectual disabilities.

\section{Design and Setting}

Qualitative study in General Practices located in NHS Greater Glasgow and Clyde, Scotland, UK.

\section{Method}

Eleven practice nurses from 11 intervention practices participated in a semi-structured interview. Analysis was guided by a framework approach.

\section{Results}

Practice nurses reported initially feeling 'swamped' and 'baffled' by the prospect of the intervention but early misgivings were not realised. Health checks were incorporated into daily routines with relative ease, but this was largely contingent on existing patient engagement. The intervention was thought most successful with patients already well known to the practice. Chronic disease management (CDM) models are commonly used by practice nurses and participants tailored health checks to existing practice. It emerged that few of the nurses utilised the breadth of the check instead modifying the check to respond to individual patients' needs. As such already recognised 'problems' or issues dominated the health check process. Engaging with the health checks in this way appeared to increase the acceptability and feasibility of the check for nurses. There was universal support for the health check ethos, though some questioned whether all adults with intellectual disabilities would access the health checks and as a consequence the long-term benefits of checks. 


\section{Conclusion}

Whilst the trial found the intervention to be dominant over standard health care, the adjustments nurses made may not have maximised potential benefits to patients. Increasing training could further improve the benefits that health checks provide for people with intellectual disabilities.

Funding: Scottish Government Change Fund and NHS Greater Glasgow and Clyde R\&D.

\section{Introduction and background}

Adults with intellectual disabilities face multiple and well-documented health inequalities (Robertson 2010). They experience premature death, some of which is preventable (Heslop et al 2013). Healthcare access for adults with intellectual disabilities has been shown to be compromised (Alborz et al 2005), and many people with intellectual disabilities depend on others recognising that they have a health need and initiating health assessments and health care on their behalf. Carers are typically relied on to act as conduits between people with intellectual disabilities and healthcare professionals to accurately relay symptoms and problems. Biologically many people with intellectual disabilities may experience increased risks associated with aspects of their underlying and other conditions, and social and environmental factors linked to poorer health outcomes such as poverty, unemployment and poor housing are common amongst adults with intellectual disabilities (Emerson et al 2009). Communication difficulties are common in adults with intellectual disabilities (Mackenzie \& Powell 2004; Purcell et al 1999).

In the United Kingdom, one policy response to such inequality has been the adoption of anticipatory care, in the form of primary care health checks. Yearly health checks were recommended by the Disability Rights Commission (Disability Rights Commission2006), and introduced as a Directly Enhanced Service in Primary Care in England and Wales in 2008. Health checks were introduced later in Northern Ireland than in England and Wales, and in parts of Northern Ireland general practices were supported in their delivery by healthcare facilitators trained in intellectual disabilities nursing. An audit of health checks in Northern Ireland reported that almost two-thirds of patients with intellectual disabilities received a health check, and particularly so in the areas that had provided healthcare facilitators, suggesting they improved uptake (McConkey et al 2015). In Scotland, annual health checks in Primary Care have been recommended by the Scottish Government's learning disabilities strategy “The Keys To Life” (The Keys to Life 2013), but not yet implemented. 
Primary Care health checks vary in scope and delivery but Lennox and Robertson (2014) have defined them as:

"the systematic gathering of a comprehensive health history that includes the person's current and past health information, and their psycho-social context. This history is reviewed by a primary health care professional, considered and clarified where necessary, and leads to a directed, systematic physical and mental health examination which results in the identification of any unmet health needs that are documented and optimally acted upon. The process optimally includes specific information about commonly missed and syndrome-specific health conditions to inform the person with intellectual disability, their care givers and the health professionals (p 195) (Lennox and Robertson 2014).

There is ample justification for adopting such a thorough anticipatory approach. Indeed adults with intellectual disability may experience more benefit than the general population where health checks have been found to be disproportionately accessed by those from higher socio-economic status groups who are at lower risk (Krogsbøll et al 2012). Adults with intellectual disabilities are infrequent primary care users, of low socio-economic status, often fail to appreciate the relevance of symptoms/problems and importantly, represent a high risk group. Moreover, health checks have been shown to offer benefits for those adults with intellectual disabilities and studies demonstrate that unmet health need was identified and, where applicable, met and maintained at follow-up (Robertson 2010, Cooper et al 2006, Buszewicz 2014). However two Australian based randomised controlled trials reported less emphatic results; one showed an increase in health promotion activity, but not a statistically significant detection of new disease (Lennox et al 2007) while the other reported no additional health benefits when the health check was augmented with a health diary (Lennox et al 2010).

Though health check studies are common, few randomised controlled trials (RCT) are available. A previous study found that checks carried out by specialist intellectual disability nurses identified mostly problems that required general medical services suggesting that health checks should be embedded within primary health care, where the ensuing investigations and interventions would be delivered (Cooper et al 2006). Practice nurses are employed in the majority of general practices across the UK (Hirst et al 1995). Their roles vary by locality and practice and individual practice needs. Although nurses have worked in general practice for many decades, their numbers and role expanded dramatically in 1990 with the introduction of a new contract in general practice (Duncan 
and Hayes 2017). This contract changed the focus of general practice from reactive to proactive and preventive. Practice nurses became largely responsible for the routine management of long term conditions and health promotion and screening. The focus on long term conditions intensified with the introduction of the Quality Outcomes Framework (Department of Health 2004) Practice nurses in the UK, therefore, routinely manage patients with long-term conditions such as asthma, diabetes and Chronic Obstructive Pulmonary Disease and as such have the potential to extend the role to adults with intellectual disabilities.

We carried out a cluster RCT design of a practice nurse delivered health check (Cooper et al 2014).Our results show that the intervention dominated standard care, being both less expensive and more effective. Alongside the trial, we conducted a nested qualitative study which sought to explore practice nurse perspectives on delivering the health check. Here we present the findings of the qualitative study.

\section{Method}

A cluster RCT was carried out in general practices in NHS Greater Glasgow and Clyde during 2011 (Cooper et al 2014). The intervention comprised of health checks undertaken by the practice nurse (with support available from intellectual disabilities nurses if requested) plus standard care while controls received standard care only. 38 general practices agreed to participate in the trial but five practices did not recruit any patients - leaving 16 in the intervention arm and 17 in the control arm. Of the 152 participants recruited to the trial, 149 ( 83 intervention; 66 control) adults with intellectual disabilities completed the trial, and intervention recipients had more new health needs identified than those in standard care.

Nested qualitative studies are an increasingly common feature of trials (Snowden 2015) and often add a level of nuance and insight untapped by regular trail methodology. In an effort to capture the perspective of the professionals delivering the trial we included a discreet qualitative study following the trial. A topic guide was prepared based of the literature and the findings of the trial. Fifteen practice nurses who had taken part in the trial were contacted by letter and invited to take part in the nested qualitative study. A series of semi-structured qualitative interviews with practice nurses participating in the trial were carried out by one researcher (LM) between March and April 2012. Interviews were face-to-face and took place at the nurses' place of work. Our overall aim for the interviews was to consider the longer term feasibility of practice nurses carrying out health checks. Nurses were asked about the practical impact of delivering the health checks including impact on workload and time, their confidence in working with this patient group, the utility and benefits of the 
check for patients and primary care, and the sustainability of the intervention. A semi-structured interview schedule allowed for consistency across interviews, but was flexible enough to allow nurses to raise issues that they felt were important. All interviews were digitally recorded with participants' permission and transcribed verbatim.

\section{Analysis}

Three researchers (MB, LM, SM) read the transcripts and engaged in a series of meetings to discuss the analytic framework and emergent themes. The framework approach (Richie and Spencer 1994) was drawn on to guide the analytic process. The approach is frequently used in health-services research and allows the original aims of the study, or a priori themes to be at the foreground of the analysis. Nevertheless, an iterative approach to data indexing was adopted and broad themes were developed into early coding frames which were discussed and agreed by three researchers (MB, LM, SM). As familiarisation with the data progressed, further iterations of the coding frames included important emergent themes. Patterns both within, and across, transcripts were identified.

Ethical approval for the trial and the nested qualitative study was obtained from MREC-Scotland A and NHS Greater Glasgow and Clyde Research and Development (10/MRE00/79).

\section{Results}

\section{Participants}

Fifteen practice nurses operating in 15 intervention practices were approached to take part and 11 of those agreed to participate. All were experienced practice nurses and none had received training in intellectual disabilities prior to the study. They conducted between one and 10 health checks each as part of the study; five nurses completed less than five health checks and six completed five or more. This is in keeping with the spread across all the participating practices. Two over-arching themes emerged from the interviews. The first centred on operational impact, as nurses discussed the extent to which the intervention impinged on their everyday workload and practice. It emerged that there was disconnect between expectations versus reality. The second major theme of engagement emerged as underpinning all aspects of the intervention and its successful adoption.

\section{Operational Impact}

For many nurses the key consideration was fitting the intervention into existing routines. Prior to the intervention, practice nurses received information to guide them through the introduction of the intervention and health check process. Though helpful, the majority of nurses emphasised the 
unwieldy volume of information and the time required to familiarise themselves with the detail. For some this was daunting, and one nurse reported feeling 'swamped' by the information, particularly when coupled with her lack of experience of working with adults with intellectual disabilities, while another regarded the information as 'a wee bit baffling'.

The time taken to carry out the check was a concern raised by nurses. Checks typically took on average 48 minutes to administer, and though few found this onerous, some did question the future feasibility of such a commitment particularly if dealing with larger numbers of patients. Time to administer the check was seen as the only adverse impact on nurses' workload, and impact was contingent upon the level of existing engagement participating patients had with the practice. Nurses reported that prior knowledge of patients and their perceived health needs eased the implementation of the health checks into existing routines and reduced the overall time of the check. Chronic disease management (CDM) was a feature of all of the nurses' roles and many saw the health check as a straightforward extension of this model, indeed for many it made the intervention more acceptable.

\section{Expectations vs. Reality}

Although there was universal agreement that the aims of the intervention were worthwhile, it was also apparent that, at the outset, many of the nurses were less than positive about their role in the process. One described being 'dumped on' and some were unclear about the rationale for their involvement. Nevertheless, all nurses reflected positively about their involvement in the intervention, in spite of initial reservations:

"There was quite a lot to do beforehand I think as I didn't really know the patients very well that I was going to see. So I was a bit swamped by it, there was a lot of information so it did mean that I knew what I was supposed to be looking for. So when I went to see them I did have quite a lot of information about them and what had been going on, which was quite good." (SR)

Later, she goes on:

"I didn't know what to expect, and I was actually a bit cheesed off when I got it as I thought this is all I need, but I thought it was good and I am glad I did it now. I was a bit anxious about it because I did not know them and I have no specialist knowledge in that area and I think it was so clear what I was supposed to be doing and I had contact with you, and I think it was good for them." (SR)

\section{Training}

Practice nurses typically saw training as optional, only to be arranged if they felt that they had specific concerns or needs. Several nurses stated that they would have preferred to have attended pre- 
organised mandatory training, despite feeling able to carry out the check adequately. Yet, what emerged was that nurses were not always entirely clear of their remit or the purpose of the health check and instead treated the questionnaire as a flexible tool. Often nurses described selecting those aspects that they deemed 'appropriate' and relevant for individual patients on a case by case basis.

\section{Engagement}

\section{Engaged Patients}

A recurring theme that underpinned all aspects of the intervention was participating patients' engagement with the practice prior to the introduction of the intervention. Organisationally, nurses reported that fore-knowledge of the patient meant a shorter and, by inference, more manageable check than those they saw less often; as one nurse reported 'it was fine, I had an easy patient'. Knowledge of the patient also shaped the check and as noted many nurses drew parallels between the intervention health check and already well-established chronic disease management models. Some eligible patients already attended chronic disease management clinics operated by the practice, and nurses felt able to select those health check areas they judged most relevant to individual patients. What emerged was an implicit tendency to place emphasis on issues they were aware of previously or target elements they were most comfortable with. Health checks amongst those, who were already being cared for within a chronic disease management model were viewed as easy opportunities.

Prior engagement with the health service also influenced nurses' perceptions of the benefits of the intervention. Most nurses assumed that engaged patients were already in receipt of optimal levels of care, and were, therefore, sceptical that any 'new' problems would emerge from the health checks. This was especially true if patients lived in supported accommodation.

"I am not sure we resolved many things going through it all. I found it fine but probably not much benefit to them because all three are already seen in the practice and are quite well known to the practice so I am not really sure they benefited. But I can see how if someone didn't attend [the practice regularly] it [the check] could open a lot of doors for them." (CL)

Despite the nurse's assertion that such patients were well cared for, the intervention health check did prompt consideration of potential disparities in the care received across patient populations. Conversely, nurses witnessed immediate benefits when those who were not well known to the practice opted to take part in the intervention. One participant acknowledged that one patient who 
was 'new' to the practice and had not previously been known to have asthma had this picked up as a result of the intervention. It was clear then that despite participant's doubts around the effectiveness of the health check in improving health there was broad agreement for the ethos and spirit of the health check. The intervention reminded practice nurses that the provision of, and access to, health services was not always evenly distributed across patient groups. Several voiced concern that those patients, they perceived to be in greatest need, may not have been reached by the intervention.

"Definitely, I think there is a wee portion that could get missed if they don't [have a check] and as I said it is obviously random but it makes you realise that they could easily be slipping through the net possibly as I don't see the rest and don't know how many patients with learning disabilities we have in the practice, I don't know if they are all getting that standard of care." (MF)

\section{Engaging with a varied patient group}

Connected to nurses' knowledge of individual patients was their confidence in dealing with individuals with intellectual disabilities. Here, the interviews expose a contradiction. Despite nurses seeing many of the patients regularly prior to the intervention and feeling comfortable managing this group, they simultaneously reported having little practical knowledge of working with individuals with intellectual disabilities. Consequently, many were unsure of, or lacked confidence around administering the check. Such uncertainty may be precipitated by the varied health needs of the patient group which struck the nurses. For example, most knew a little about Down's syndrome but little else and the intervention offered ideal learning opportunities [Box 1 a]. Across the interviews there were a handful of examples that illustrated that a lack of knowledge and experience may impact on care. One nurse was not clear initially whether the 'exercise on referral' scheme was open to 'this group', another questioned the extent to which she should 'believe' what patients told her and it was evident communication more generally could be problematic [Box $1 \mathrm{~b}]$ :

Box 1Engaging with a varied patient group 
a "I was interested in the bits like they were highlighting how much they miss gastroesophageal reflux disease in people and there were questions on that that were linked. So yes I think they were fairly standard but there was a couple of wee surprises that I hadn't realised how much of a problem that can be." (RA)

$B$ "Yes sometimes I am not exactly sure whether they were able to express what they really mean and understand my questions properly. I know there had been different ways you can put questions to patients to make them understand it a wee bit more and some of the answers was maybe wrong to say you don't want to hear it as you don't know if it's true. Like there was one boy who said his family were not nice to him because he was told you need to calm down here or whether he was justified and they were being unfair with him, things like that." (CL)

$c$ "There was one in particular, a young man with mild learning disabilities, but in a sense a more difficult consultation as his mum came in first to discuss particular areas of care that she was concerned about in relation to her son. One of those was that he was using cannabis and there was a problem with his asthma ...... So I spent quite a bit of time with him. That was very different to the others because a lot of them had come with more severe disabilities and like I said I found them much more straight forward and easier in some ways which you would have thought had been the other way round would have been easier than the person that lives on his own with his mum and had a bit of a chaotic lifestyle."(JF)

The extract raises an apparent tension inherent in the intervention process. It was evident that nurses felt that checks with patients with more severe difficulties, while sometimes demanding physically, were easier to complete than those with 'milder' patients who often proved more challenging [Box $1 c]$.

\section{Discussion}




\section{Summary of main findings and comparison with existing literature}

Although previous studies have explored the perceptions of GPs carrying out health checks for adults with intellectual disabilities, this is the first study that reports on practice nurses' views on providing health checks. Perhaps unsurprisingly given the additional commitment that the health check intervention required, practice nurses reported feeling apprehensive both about the information they had to digest before the check and the impact on their already busy workload. Time pressures might explain why none of the nurses accessed training despite simultaneously reporting that they might benefit from training. Nevertheless, when nurses reflected on the health check process their initial concerns were not realised. Such gaps between expectations and reality echo findings from a previous RCT that reported GPs perceptions of undertaking a health check for adults with intellectual disabilities which found that GPs views were more positive post-intervention (Lennox et al 2013). In our study, as in that of Lennox and colleagues, changes post-intervention could in part be explained by previous knowledge of their patients. Practice nurses describe relying on their chronic disease management (CDM) skills when carrying out the checks and adopting a CDM approach assisted the transition of the health check into pre-existing routines. The foregrounding of the CDM model by practice nurses is reflected in the findings from the wider trial which found that practice nurses recorded QOF items regularly, while other health needs were less frequently identified. This is also consistent with practice nurses' perception that participating patients were already in receipt of optimal care. Data from the wider trial, however, do not support this. Practices in the health check group significantly increased their completion of QOF indicators during the trial (Cooper et al 2014). Nurses' prior knowledge of patients, their ease with CDM models and their uncertainty about some aspects of the health check resulted in an adapted health check with CDM at its core. This is despite the different pattern of health needs that people with learning disabilities have, compared with the general population, which are not reflected in the QOF.

Relying on the CDM confirms practice nurses' prediction that the move towards QOF targets in primary care may stifle nurses' frames of reference when dealing with non-QOF issues (MacGregor et al 2008). This finding arguably questions general nurses' ability to undertake health checks with specialist groups of patients and indeed this approach was less effective than a previous study where health checks were undertaken by specialist intellectual disability nurses (Cooper et al 2006). When screening for depression within chronic disease management encounters, practice nurses report a tendency to drift towards 'tick-boxing' (Maxwell et al 2013). Yet, when specialist nurses do undertake general health checks for specialist populations, they report lacking confidence in dealing with more general health issues (Robson et al 2012). More concerning however is that reliance on what is already 
known may obscure additional health needs which may continue to be unidentified and unmet. While the presence of practice nurses improves quality in primary care (Griffiths et al 2011) there is a risk that continually adding routine tasks to their remit may result in both reduced quality and de-skilling (McDonald et al 2009). Finally, nurses' recognise that a hard to reach group may remain unengaged with primary care and so fail to benefit from the intervention. Such disparity ultimately serves to exacerbate rather than ameliorate inequalities in a multiply disadvantaged population ( $O^{\prime}$ Donnell et al 2012).

We believe there have only been two controlled trials on health checks for people with intellectual disabilities conducted within the UK health-care system. Our trial demonstrated that practice nurse health checks were clinically and cost effective compared with standard care. However, the extent of benefit was less than that found in a previous (non-randomised) controlled trial in which health checks were conducted by intellectual disabilities nurses, although they took five times longer to conduct each health check. The study with intellectual disabilities nurses also found the health checks to be inexpensive, and indeed, the mean cost of care for the adults who received standard care only was greater than for the adults who received the health check intervention (Romeo et al 2009). Our study has demonstrated that practice nurses felt they were able to accommodate the health checks into routines, but considered that there should have been mandatory training prior to undertaking them: a future study should address the added value of mandatory training. The different methodological design of both these trials (randomised versus non-randomised), and the different perspectives taken in the two economic evaluations renders direct comparisons difficult, so it is unclear which model of delivery - intellectual disabilities nurses, or practice nurses - is the best. A future clinical and cost effectiveness trial to compare between the two types of health check delivery might indicate which method is the most effective.

\section{Strengths and Limitations}

Our paper reports findings from the nested qualitative study within the wider RCT, the first to report outcomes related to practice nurse delivered health checks in primary care. The findings add explanation to the results from the wider study and in particular demonstrate that practice nurses, familiar with QOF items and CDM concentrated on those within the health checks. Although the qualitative component was small and our analysis is based on 11 practice nurse interviews we were able to recruit nurses in the majority of intervention practices. Given that newly detected health needs were identified in both intervention and control arms, interviews with practice nurses in the control 
arm would provide an additional useful dimension to the findings. On reflection, adopting a theory driven approach to the study, such as Normalisation Process Theory (May and Finch 2009, May et al 2011). ' which considers how interventions become routine may have elicited more in-depth accounts from practice nurses.

\section{Conclusions}

Despite initial misgivings practice nurses engaged with the intervention and, although they tended to focus on those areas they were comfortable with, such as chronic disease management, the overall trial results show that general health significantly improved for those in the intervention group. Nevertheless, the success of the intervention from the nurses' perspective was often contingent on already engaged patients with previously identified health needs, therefore suggesting that the hardest to engage patients, who arguably may be most in need, may have benefitted less from the intervention. Indeed if practice nurses conduct health checks within their existing 'comfort zones' some important health needs may be missed. The nurses identified the need for mandatory training prior to completing the health checks, despite little availing of the optional training that was available. To date, there has been insufficient research conducted on this topic to recommend whether health check delivery by practice nurses or by intellectual disabilities nurses is the most clinically and cost effective: any future comparison trial should additionally consider incorporating mandatory training prior to undertaking the health checks.

\section{References}

Alborz A, McNally R, Glendinning C. Access to health care for people with learning disabilities in the UK: mapping the issues and reviewing the evidence. J Health Serv Res Policy 2005;10:173-82. doi: $\underline{10.1258 / 1355819054338997}$

Buszewicz, M., Welch, C., Horsfall, L., Nazareth I, Osborn D, Hassiotis A et al Evaluation of a national scheme incentivising General Practitioners to provide annual health checks to patients with intellectual disability. Lancet Psychiatry, 2014;1(7) 522-530. https://doi.org/10.1016/S2215$\underline{0366(14) 00079-0}$

Cooper S-A, Morrison J, Melville C, Finlayson J, Allan L, Martin G et al. Improving the health of people with intellectual disabilities: outcomes of a health screening programme after one year. JIDR 2006; 50: 667-77. DOI: 
Cooper, S-A., Morrison, J., Allan, L., McConnachie A, Greenlaw N, Melville C, et al. Practice nurse health checks for adults with intellectual disabilities: randomised controlled trial. Lancet Psychiatry, 20141(7) 511-521 http://dx.doi.org/10.1016/S2215-0366(14)00078-9

Department of Health. QOF guidance. London: Department of Health, 2004. http://www.dh.gov.uk/en/Policyandguidance/

Organisationpolicy/Primarycare/Primarycarecontracting/QOF/ DH_4125653

Disability Rights Commission (2006) Equal treatment: closing the gap. Health formal investigation report. London: Disability Rights Commission.

Duncan D, Hayes S. Developing the role of the GP nurse. Independent Nurse. Published $15^{\text {th }}$ of May 2017. http://www.independentnurse.co.uk/professional-article/developing-the-role-of-the-gpnurse/155185/

Emerson E, Madden R, Robertson J, et al. Intellectual and Physical Disability, Social Mobility, Social Inclusion \& Health: Background paper for the Marmot Review. Lancaster: Centre for Disability Research, Lancaster University, 2009.

Griffiths P, Maben J, \& Murrells T. Organisational quality, nurse staffing and the quality of chronic disease management in primary care: observational study using routinely collected data. Int J Nurs Stud. 2011 Oct;48(10):1199-210. doi: 10.1016/j.ijnurstu.2011.03.011

Heslop, P., Blair, P., Fleming, P., et al (2013) Confidential Inquiry into premature deaths of people with learning disabilities (CIPOLD). University of Bristol.

Hirst $\mathrm{M}$, Atkin $\mathrm{K}$, Lunt $\mathrm{N}$ Variations in practice nursing: implications for family health services authorities Health Soc Care Community 1995;3(2);83-97. DOI: 10.1111/j.1365-2524.1995.tb00009.x 
Krogsbø|l LT, Jørgensen KT, Larson CG, and Gotzsche P. General health checks in adults for reducing morbidity and mortality from disease. Cochrane systematic review and meta-analysis. BMJ 2012; 345: e7191. doi: https://doi.org/10.1136/bmj.e7191

Lennox, N G. and Robertson, J (2014). Health checks. In Laurence Taggart and Wendy Cousins (Ed.), Health promotion for people with intellectual and developmental disabilities Maidenhead, United Kingdom: Open University Press.

Lennox N, Bain C, Rey-Conde T, et al. Effects of a comprehensive health assessment programme for Australian adults with intellectual disability: a cluster randomized trial. Int J Epidemiol 2007; 36: 13946. https://doi.org/10.1093/ije/dyl254

Lennox N, Bain C, Rey-Conde T, et al. Cluster randomized controlled trial of a tailored intervention to improve health for adults with intellectual disability who live in private dwellings. J Applied Res Intellect Disabil 2010; 23: 303-11.

Lennox, N. G., Brolan, C. E., et al. General practitioners' views on perceived and actual gains, benefits and barriers associated with the implementation of an Australian health assessment for people with intellectual disability. JIDR 201357 10: 913-922. doi:10.1111/j.1365-2788.2012.01586.x

Maxwell M, Harris FM, Hibberd C, Donaghy E, Pratt R, Williams C et al A qualitative study of primary care professionals' views of case finding for depression in patients with diabetes or coronary heart disease in the UK (2013)BMC Family Practice 2013, 14:46 doi:10.1186/1471-2296$14-46$

May C, and Finch T Implementing, embedding, and integrating practices: an outline of Normalization Process Theory. Sociology 2009 43: 535-554.

May C, Finch T, Ballini L, et al Evaluating complex interventions and health technologies using normalization process theory development of a simplified approach and web-enabled toolkit. $B M C$ Health Serv Res 2011 11: 245. https://doi.org/10.1186/1472-6963-11-245

McConkey R, Taggart L, Kane M Optimizing the uptake of health checks for people with intellectual disabilities. Jnl of Intellectual Disabilities 2015;19 (3) 205-214 doi.org/10.1177/1744629514568437 
McDonald R, Campbell S, \& Lester H, Practice nurses and the effects of the new general practitioner contract in the English National Health Service: The extension of a professional project? Soc Sci Med 2009 68, (ㄱ), doi: 10.1016/j.socscimed.2009.01.039.

McGregor, W., Jabareen, H., O'Donnell, Cet al. Impact of the 2004 GMS contract on practice nurses: A qualitative study. BJGP 2008 , 58, 711-719. doi: 10.3399/bjgp08X342183.

McKenzie K, Powell H. Health screening. Learning Disability Practice 2004;7(10):34-38.

O'Donnell, C., Mackenzie, M., Reid, M., Turner F, Clark J, Wang Y. et al Delivering a national programme of anticipatory care in primary care: a qualitative study. BJGP 2012 62(597). doi: 10.3399/bjgp12X636137.

Purcell M, Morris I, McConkey R. Staff perceptions of the communicative competence of adult persons with intellectual disabilities. British Journal of Developmental Disabilities 1999;45:16-25. DOI: $10.1179 / 096979599799155957$

Ritchie J, Spencer L (1994) Qualitative data analysis for applied policy research. In: Bryman A, Burgess R, editors. Analysing Qualitative Data. London: Routledge.

Robertson, J., Roberts, H., \& Emerson, E. (2010). Health checks for people with learning disabilities: Systematic review of impact. Durham, UK: Improving Health and Lives: Learning Disabilities Observatory.

Robson, D., Haddad, M., Gray, R., et al.. Mental health nursing and physical health care: A crosssectional study of nurses' attitudes, practice, and perceived training needs for the physical health care of people with severe mental illness. Int J Ment Health Nurs2012. doi: 10.1111/j.14470349.2012.00883.x.

Romeo, R., Knapp, M., Morrison, J., Melville, C., Allan, L., Finlayson, J., Cooper, S-A. (2009) Cost estimation of a health screening intervention for people with intellectual disabilities in the UK. Journal of Intellectual Disability Research, 53, 426-439.

Snowdon C Qualitative and mixed methods research in trials Trials 201516:558 https://doi.org/10.1186/s13063-015-1084-4 
